\title{
Nécropole Santa Rosa (Rome, Cité du Vatican)
}

Henri Duday, Carla Caldarini, Stefania Di Giannantonio, Giandomenico Spinola, Leonardo Di Blasi et Monica Ricciardi

\section{(2) OpenEdition Journals}

\section{Édition électronique}

URL : http://journals.openedition.org/cefr/1104

DOI : $10.4000 /$ cefr. 1104

ISSN : 2282-5703

Éditeur

École française de Rome

Référence électronique

Henri Duday, Carla Caldarini, Stefania Di Giannantonio, Giandomenico Spinola, Leonardo Di Blasi et Monica Ricciardi, « Nécropole Santa Rosa (Rome, Cité du Vatican) », Chronique des activités archéologiques de l'École française de Rome [En ligne], Italie centrale, mis en ligne le 20 février 2014, consulté le 15 novembre 2019. URL : http://journals.openedition.org/cefr/1104; DOI : 10.4000/cefr. 1104

Ce document a été généré automatiquement le 15 novembre 2019.

(c) École française de Rome 


\title{
Nécropole Santa Rosa (Rome, Cité du Vatican)
}

\author{
Henri Duday, Carla Caldarini, Stefania Di Giannantonio, Giandomenico \\ Spinola, Leonardo Di Blasi et Monica Ricciardi
}

\section{NOTE DE L'ÉDITEUR}

Ce texte est rédigé par Henri Duday. L'analyse anthropologique est assurée par H. Duday, C. Calderini et S. Di Giannantonio ; l'analyse archéologique par G. Spinola, L. Di Blasi et M. Ricciardi.

1 L'année 2013 a vu se poursuivre les recherches anthropologiques entreprises depuis novembre 2011 dans cette portion de la vaste nécropole romaine qui longeait la Via Triumphalis, dont la fouille s'est déroulée dans un contexte d'archéologie préventive ${ }^{1}$. Ces travaux s'inscrivent dans le programme d'une convention quadripartite qui a été signée au printemps 2012 entre la Direction des Musei Vaticani, l'École française de Rome, l'Université Bordeaux 1 (UMR 5199, Laboratoire d'Anthropologie) et la Soprintendenza archeologica di Roma.

2 Deux campagnes d'investigations ont été réalisées en 2013, du 29 avril au 11 mai et du 8 au 24 septembre.

\section{Le mausolée $X X$}

3 Nous avons longuement détaillé dans la précédente Chronique des activités archéologiques de l'École française de Rome les résultats concernant le premier des mausolées que nous avons étudiés (mausolée XX). De fait, nous avions prévu d'explorer en 2013 le petit coffre maçonné qui se trouve sous le monument, coffre auquel donnent accès d'une part un conduit vertical parementé d'imbrices affrontés, et d'autre part une tubulure cylindrique en céramique (conduit à libations). Ce caisson rectangulaire a des parois verticales formées d'au moins cinq assises de briques, sa couverture est formée de deux tuiles plates 
disposées en bâtière, avec deux autres tuiles plates verticales aux extrémités ; le fond se trouve environ $140 \mathrm{~cm}$ au-dessous du sol de tuileau du mausolée. Quelques prélèvements ont été effectués par aspiration dans le remplissage de terre et de gravats provenant de la voûte du mausolée; ils ont livré des os humains brûlés se rapportant à un adulte de robustesse moyenne, celui-ci ne pouvant en aucune manière être l'un des quatre adultes (une femme, un homme et deux sujets de sexe indéterminé) dont les os étaient contenus dans les urnes de céramique étudiées en 2012. Bien que ces premiers résultats soient prometteurs, nous avons préféré surseoir à la poursuite de l'opération. La fouille méthodique et précise nécessitera en effet l'utilisation d'un système de télémanipulation (bras articulé) associé à une caméra endoscopique.

4 Il est possible que le défunt dont les restes brûlés se trouvent dans la petite chambre souterraine soit Alcimus, l'esclave de Néron pour qui son épouse Fabia Philtate a fait ériger le tombeau. Cela reste cependant à confirmer par la fouille exhaustive de ce compartiment.

\section{Le mausolée XXXIV}

La campagne 2013 nous a également permis d'achever l'étude du mausolée XXXIV dont le dédicataire n'est pas connu (absence de stèle).

Fig. 1 - Nécropole Santa Rosa. Vue du mausolée XXXIV en cours d'étude.

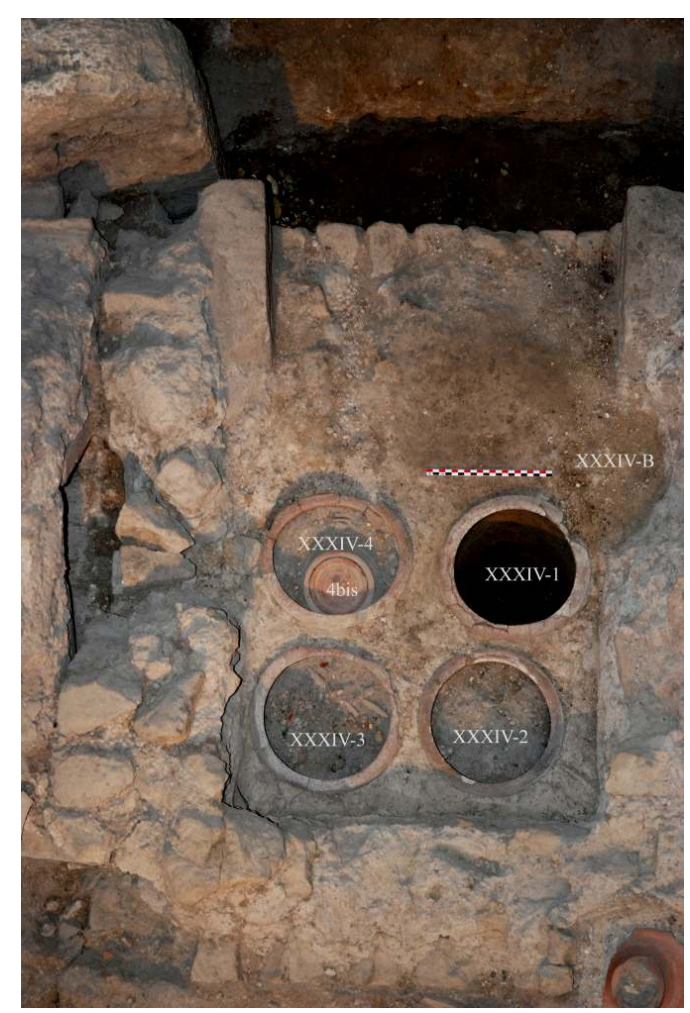

Le contenu de l'urne XXXIV-1 a été prélevé, le remplissage des trois autres urnes est encore en place. Dans I'urne XXXIV-4, on voit le sommet de la petite urne XXXIV-4bis avec son couvercle.

Photographie H. Duday. 


\section{Les sépultures de l'US 111 , deux sépultures secondaires à crémation postérieures à l'abandon du mausolée}

Lors de la fouille de ce secteur, en mai 2003, deux ensembles d'ossements humains avaient été prélevés dans l'US 111, postérieure à l'abandon du mausolée. Il n'y avait pas d'urne en céramique mais le caractère parfaitement circonscrit des deux dépôts, de plan circulaire, suggère que les os étaient peut-être contenus dans un réceptacle en matière organique (tissu?).

7 La sépulture XXXIV/US 111 - B a en partie recoupé la paroi maçonnée sud-ouest de la chambre; le creusement s'arrête sensiblement sur le sol de tuileau, il a légèrement endommagé la lèvre de l'urne XXXIV-1 prise dans celui-ci. La fouille a livré 1160,2 g d'os humains brûlés qui se rapportent à un adulte probablement de sexe masculin (grande incisure ischiatique fermée). La crémation est dans l'ensemble assez peu poussée.

La sépulture XXXIX/US 111 - C se trouve à l'extérieur du mausolée, à gauche de l'entrée. Les résultats sont moins probants dans la mesure où elle n'a été que partiellement fouillée : la partie inférieure du dépôt cendreux avec de nombreux fragments osseux est encore en place dans la nécropole. Notre étude n'a donc porté que sur une partie du dépôt : 467,8 g d'os humains brûlés, qui se rapportent à un adulte de robustesse moyenne, de sexe indéterminé.

\section{Les urnes du mausolée XXXIV}

Quatre grandes urnes en céramique de dimensions sensiblement égales sont prises dans la maçonnerie du mausolée. Leur lèvre affleure à la surface du sol maçonné. L'hypothèse initiale était naturellement qu'il y avait là quatre sépultures à crémation. La fouille a démontré qu'il n'en était rien, l'ensemble du dispositif correspondant à la tombe d'un seul et même individu.

10 L'urne cinéraire proprement dite est l'urne XXXIV - 3 située dans l'angle nord de la chambre. Elle contenait les restes brûlés d'un adulte sans doute relativement âgé (pathologie dégénérative évoluée). Il s'agit indiscutablement d'un homme (grande incisure ischiatique très fermée). La masse totale des os humains est très élevée (1967,2 g), la crémation est irrégulière mais dans l'ensemble assez peu poussée. La fragmentation des os est forte, avec un poids moyen sauf esquilles très faible $(0,70 \mathrm{~g})$.

11 Les urnes XXXV - 1 (angle sud de la chambre) et XXXIV - 2 (angle ouest) n'ont quand à elles livré qu'une faible quantité d'os humains brûlés ( $80,5 \mathrm{~g}$ et $50,1 \mathrm{~g}$ respectivement). Ils étaient mêlés à des cendres riches en charbons de bois qui occupaient la moitié supérieure du remplissage des urnes. Les paramètres ostéologiques présentent toutes les caractéristiques d'un curage de l'aire de crémation réalisé après la collecte des morceaux les plus gros, tels que nous avons pu les définir pour la nécropole de la Porta Nocera à Pompéi $^{2}$ : un poids moyen sauf esquilles très abaissé (respectivement $0,32 \mathrm{~g}$ et $0,19 \mathrm{~g}$ ), avec au contraire un indice pondéral des mains élevé (3,6 et 2,6 pour une valeur de référence de 2,5, alors que le même indice n'est que de 0,9 dans l'urne XXXIV - 3). L'urne XXXIV - 4 (angle est de la chambre) contenait une quantité infime de fragments humains brûlés $(6,8 \mathrm{~g})$, de même que la petite urne fermée par un couvercle (urne XXXIV - 4bis) qui avait été placée à l'intérieur ( $1,5 \mathrm{~g}$ seulement). 

trouvent principalement dans l'urne XXXIV-3: nous avons en effet pu trouver des remontages absolument certains d'une part entre deux fragments de crâne au niveau d'une suture (XXXIV - 1 R05 et XXXIV - 3 R04 Est) et d'autre part entre deux fragments jointifs d'un os du carpe, la capitatum gauche (XXXIV-1, R05 et XXXIV-3, R08). De même, il est acquis que l'urne XXXIV - 2 contient les restes du même sujet (collage entre deux fragments jointifs d'une vertèbre thoracique (XXXIV - 2, R01 et XXXIV - 3, R09). Il existe de même une liaison presque certaine entre deux fragments de la diaphyse d'un os long du membre supérieur pathologique provenant l'un de l'urne XXXIV - 3 (R05 Est) et l'autre de l'urne XXXIV - 4bis (R03).

fragments de cruches en céramique, de vases à paroi fine, brûle-parfum, monnaie de bronze, clous en fer, balsamaires de verre...). Comme pour les os humains, des remontages ont pu être établis entre les tessons d'une cruche (urceolus) qui proviennent de différentes urnes (XXXIV - 1, 2 et 4). Des liaisons identiques ont été trouvées entre plusieurs tessons d'un gobelet à paroi fine.

Il est donc acquis que le mausolée XXXIV est bien une tombe individuelle, exclusivement destinée à un homme adulte assez âgé. De l'urne XXXIV - 3 (R02 Est) provient cependant le germe d'une molaire permanente (probablement la deuxième molaire inférieure droite) dont la couronne est complète mais dont les racines n'étaient pas encore formées au moment du décès; il s'agit donc d'un sujet immature, dont l'âge peut être très approximativement estimé à 7 ou 8 ans (si l'identification de la dent est exacte). Par ailleurs, l'urne XXXIV-2 (R03) a livré un fragment antérieur de temporal droit incompatible avec un fragment équivalent qui provient de l'urne XXXIV - 3 (R07), celui-ci étant le symétrique certain du fragment gauche XXXIV - 3 (R05 Est). Le fragment surnuméraire trouvé dans l'urne XXXIV - 2 pourrait éventuellement appartenir à l'enfant dont la présence est attestée par le germe de molaire. Ces deux vestiges ont probablement été ramassés par erreur lors de la collecte, témoins résiduels d'une (ou de deux) crémation(s) qui aurai(en)t été pratiquée(s) antérieurement au même emplacement.

\section{L'urne de Stlaccia Helpis}

15 Une vitrine de l'exposition présente une petite urne de marbre (numéro d'inventaire 52165) richement décorée, sur laquelle un cartouche indique qu'elle contient les restes de Stlaccia Helpis, morte à l'âge de 33 ans.

Les os brûlés qu'elle contenait se rapportent effectivement à un sujet adulte extrêmement gracile; les fragments conservés des os coxaux ne permettent pas de proposer une diagnose sexuelle, mais on notera que les corps des vertèbres thoraciques sont vraiment de très petites dimensions. L'absence de toute lésion dégénérative suggère qu'il s'agit d'un sujet relativement jeune. Sans être probantes, ces constatations sont donc parfaitement compatibles avec l'identité qu'indique l'inscription.

17 Les os sont pris dans une matière blanche très compacte qui s'est révélé être un mélange de deux carbonates de calcium, la calcite et l'aragonite. Il est pour l'instant difficile d'expliquer comment cette matière s'est accumulée dans l'urne (et exclusivement à l'intérieur de l'urne) et a ennoyé la totalité du dépôt osseux. Lors de la fouille, cette urne 
a malheureusement été découverte en situation secondaire, hors de tout un contexte monumental.

La masse totale des os brûlés est de 845,2 g, mais elle est forcément surévaluée en raison de l'incrustation minérale qui n'a pu être complètement enlevée.

\section{Le mausolée III}

19 Le mausolée III présente un très riche décor de stucs qui été récemment restauré. L'entrée se fait par une porte ouverte au sud. Ses parois gauche (ouest), de fond (nord) et droite (est) sont creusées de niches de plan rectangulaire ou semi-circulaire. Ces niches sont réparties sur trois niveaux pour les parois ouest et est, alors qu'il y a seulement deux niveaux pour la paroi nord, l'étage inférieur étant occupé par une loge sous arcosolium devant l'entrée de laquelle se trouvait une amphore intacte. Le sol du mausolée est mosaïqué.

La plupart des niches contiennent une ou deux urnes prises dans la maçonnerie. Il y en a au total 12 pour le niveau inférieur ( 6 à gauche et 6 à droite), 16 pour l'étage moyen (6 à gauche, 4 au fond et 6 à droite) et 3 pour l'étage supérieur (2 à gauche et une à droite), soit au total 31 urnes en place. Des urnes « libres» (non engagées dans la construction) étaient posées sur le fond de quelques niches : une dans la niche d'entrée du côté ouest, à l'étage inférieur (niche 1, urne III -1), une dans la niche de fond du côté ouest, à l'étage moyen (niche 9, urne III - 2), une dans la niche d'entrée du côté ouest, à l'étage supérieur (niche 17, urne III - 35), enfin une dans la niche de fond du côté ouest, à l'étage supérieur (niche 18, urne III - 34). Les urnes III - 1 et III - 2 sont présentées dans une des vitrines d'exposition, avec les os brûlés qu'elles contenaient, l'urne III - 35 avait elle aussi été prélevée lors de la fouille, l'urne III - 34 est encore en place dans la niche où elle a été déposée. Les photographies et les notes prises au moment de la fouille ont permis de retrouver leur situation originelle.

21 La campagne 2013 a été principalement consacrée à l'étude anthropologique des vestiges qui avaient été récupérés lors du dégagement du monument. De nombreux lots d'ossements brûlés ont en effet été mis au jour dans les différentes niches; leur provenance est indiquée de manière plus ou moins précise. Dans la plupart des cas, les os n'étaient pas dans des urnes; la disposition de certains ensembles, en amas délimités, indique qu'ils devaient être contenus dans des enveloppes périssables (sacs en tissu ou linges repliés, coffrets de bois?). D'autres lots d'ossements proviennent des US définies dans la partie centrale de la chambre. Certains étaient dans des urnes basculées, qui à l'origine devaient être posées dans les niches des parois, d'autres formaient des ensembles plus diffus. Nous donnerons simplement ici un bref aperçu des principaux résultats que nous avons obtenus pour chacun de ces lots. Les valeurs des principaux paramètres quantitatifs sont regroupées dans le tableau 1.

\section{Les niches de la paroi gauche (ouest), dépôts en urne}

Urne III - 1 (niche 1) : un seul sujet, un adolescent robuste de sexe indéterminé.

Urne III - 2 (niche 9) : un seul sujet, un adulte très gracile de sexe indéterminé.

Urne III - 35 (niche 17) : les vestiges se rapportent à un adulte de sexe indéterminé. Il s'agit de fragments osseux de petites à très petites dimensions; ils étaient mêlés à un 
sédiment pulvérulent riche en charbons de bois et macrorestes végétaux (pignons de pin, graines de micocoulier). De fait, ce petit dépôt présente toutes les caractéristiques d'un curage partiel d'aire de crémation, avec notamment une surreprésentation des phalanges de la main ( 5 fragments sur un total de 6 pièces osseuses identifiées pour les membres supérieurs!).

\section{Les niches de la paroi ouest, dépôts non contenus dans des urnes}

III - Niches 10 et 11 (paroi gauche au fond, étage moyen et paroi de fond, à gauche, étage moyen - le prélèvement a été fait sans distinction du contenu de chacune des niches) : la masse totale est considérable. L'ensemble correspond à au moins 6 sujets (4 adultes dont un homme, les autres étant de sexe indéterminé, un adolescent et un grand enfant ou adolescent de moins de 16 ans). De nombreuses lésions pathologiques ont été relevées : arthrose cervicale (3 vertèbres), thoracique (11 vertèbres, avec deux blocs bivertébraux acquis) lombaire (8 vertèbres); aspect dégénératif de l'incisure claviculaire sur un manubrium sternal ; deux sujets présentent une arthrose sévère du coude (remaniements de la cupule radiale avec éburnation) ; enthésopathies multiples (une tubérosité bicipitale de radius, crête iliaque, deux ischiums symétriques, patella, insertion du muscle soléaire sur deux tibias, insertion du tendon d'Achille sur un calcanéus).

III - Niches 8 et 17 (paroi gauche, niches côté entrée des étages moyen et supérieur) : un adulte de robustesse moyenne, de sexe indéterminé ; les fragments contenus dans l'urne III - 35 (niche 17) n'appartiennent pas à cet individu.

\section{Les niches de la paroi droite (est), dépôts non contenus dans des urnes}

III - Niche 7 (paroi droite, niche centrale de l'étage inférieur) : 3 sujets représentés, un adulte, un grand adolescent ou adulte jeune et un enfant de 7 à 10 ans. A noter les séquelles d'une fracture de côte et des lésions d'arthrose sur l'extrémité distale d'un radius droit.

III - Niche 14 ? (paroi droite, niche au fond, étage moyen) : la quantité d'os brûlés est très faible $(37,3 \mathrm{~g})$; ils se rapportent à un sujet de taille adulte (adulte ou adolescent) de sexe indéterminé. La localisation de ce dépôt est incertaine.

9 III - Niche 15 (paroi droite, niche centrale de l'étage moyen) : quelques fragments brûlés $(56,3 \mathrm{~g})$ appartiennent à un sujet de taille adulte (adulte ou adolescent) de sexe indéterminé. Il y a aussi une clavicule gauche, une côte et la partie distale d'une diaphyse tibiale non brûlées qui se rapportent à un enfant relativement jeune (il pourrait s'agir du même sujet que celui qui est représenté dans la niche III - 19 ( $c$ f. infra).

III - Niche 16 (paroi droite, niche de l'étage moyen située près de l'entrée) : ici encore, il n'y a que très peu d'os ( $41,3 \mathrm{~g}$ de fragments brûlés). Ils indiquent la présence d'un adulte robuste, de sexe indéterminé.

III - Niche 20 (paroi droite, niche de l'étage supérieur située près du fond) : les os brûlés qui constituent ce dépôt relativement important appartiennent en fait à trois sujets : un adulte robuste relativement âgé (pathologie dégénérative de la colonne vertébrale, sutures crâniennes en cours de synostose) et de sexe indéterminé, un adulte jeune ou grand adolescent, enfin un grand enfant dont l'âge peut être estimé entre 10 et 14 ans. 
L'adulte âgé est le mieux représenté, mais la présence des deux autres sujets n'est certainement pas fortuite. Lorsque des os brûlés résiduels sont ramassés "par erreur " sur une aire de crémation qui a été utilisée antérieurement, le sujet surnuméraire n'est en général attesté que par de rares fragments de petite taille, les morceaux les plus gros ayant été prélevés immédiatement après la crémation pour être déposés dans la tombe. Ici, l'adolescent ou adulte jeune est représenté au moins par un fragment du temporal droit (processus mastoïdien), un corps de vertèbre thoracique, un fragment de clavicule et une diaphyse humérale; quant à l'enfant, il a été identifié le corps d'une vertèbre lombaire (la cinquième) et les portions proximales du radius droit et de l'ulna gauche.

\section{Les niches de la paroi du fond de la chambre (paroi nord)}

Les os brûlés trouvés dans la niche III - 11 (paroi du fond, niche gauche de l'étage moyen) ont été prélevés en même temps que ceux de la niche la plus proche (niche III - 10, paroi gauche, niche de fond de l'étage moyen). Ils ont donc été décrits comme un ensemble commun, III - Niches 10 et 11 (cf. supra).

III - Niche 19 (paroi du fond, niche droite de l'étage supérieur) : les fragments brûlés $(46,3 \mathrm{~g}$ au total) correspondent à un adulte apparemment très robuste, de sexe indéterminé. Il y a également quelques os non brûlés: une vertèbre lombaire et un fragment d'os coxal appartenant à un adulte robuste, le corps d'une vertèbre thoracique de grand enfant ou adolescent (listel non soudé, mais dimensions comparables à celles d'une vertèbre d'adulte) et les deux maxillaires d'un enfant d'environ 6 ans. Bien qu'il n'y ait donc que très peu d'os non brûlés, ils indiquent la présence de trois sujets.

\section{Les restes humains trouvés dans la partie centrale du mausolée}

III - «Olle in US $110 »$ : la salle centrale était comblée par des sédiments fortement perturbés par les spoliations tardives dont le mausolée a été victime. Plusieurs urnes (au moins trois) ont été trouvées à proximité de la paroi ouest. Le lot d'ossements brûlés accompagné de l'étiquette "Olle in US 110 » provient très probablement de l'une de ces urnes. Il correspond à un seul et même sujet, un adulte de sexe indéterminé. On signalera des lésions d'arthrose sévère sur une vertèbre cervicale et quelques spicules enthésopathiques au niveau de la crête iliaque.

Il y avait également deux volumineux sacs contenant des os humains brûlés qui portaient des indications similaires: "SR03. Sepolcro III-ossa combuste». Ils ont été analysés séparément et comme il n'a été trouvé aucune liaison entre les contenus des deux sacs (fragments osseux ou tessons de céramique), nous avons traité séparément le contenu des deux sacs. Nous les désignerons arbitrairement comme étant les ensembles III - A et III - B.

III - A : la masse totale des os humains brûlés est relativement faible $(976,7 \mathrm{~g})$. Elle correspond cependant à trois sujets, deux adultes, l'un robuste et l'autre gracile, tous deux de sexe indéterminé, et un grand enfant (listels vertébral non soudé, épiphyse distale d'un métacarpien non soudée).

III - B : ici encore, les os humains brûlés représentent une masse assez faible $(873,4 \mathrm{~g})$, mais ils indiquent la présence de deux adultes, un homme et un sujet de sexe indéterminé. L'un est relativement âgé (pathologie dégénérative aux différents étages de la colonne vertébrale, enthésopathies de la crête iliaque) ; une tête humérale a un aspect 
anormal, probablement lié à une luxation invétérée de l'épaule. L'autre sujet est décédé au cours de la troisième décennie (épiphyse médiale de la clavicule en cours de synostose).

\section{Le contenu de l'urne III - 22}

La campagne d'étude du mausolée III s'est poursuivie par la fouille du contenu de l'une des 31 urnes prises dans la maçonnerie, l'urne III -22. Elle se trouve dans la niche centrale de la paroi de fond, à l'étage moyen (niche 12), qui a été très endommagée par une tranchée tardive de spoliation. Le démontage du contenu de l'urne a été réalisé par passes consécutives de 2 à $3 \mathrm{~cm}$ d'épaisseur (R01 à R08), selon un protocole désormais éprouvé (Van Andringa et al., 2013, p. 5-16). Au fond se trouvait une couche compacte de fragments de mortier, épaisse de $9 \mathrm{~cm}$, sur laquelle étaient posés trois clous en fer, l'ensemble étant surmonté par le dépôt d'os humains brûlés.

La masse totale des os brûlés est faible $(1214,4 \mathrm{~g})$, avec une fragmentation forte (le poids moyen des os sauf esquilles est de $0,51 \mathrm{~g}$ ). Ils se rapportent à un adulte peut-être de sexe féminin ; la portion conservée de l'incisure ischiatique, qui semble ouverte, est cependant trop courte pour que la détermination du sexe soit absolument certaine.

En surface reposait un fragment de temporal gauche (pétreux) de couleur noire (crémation peu poussée), qui se rapporte à un sujet robuste. Il s'agit d'une pièce surnuméraire, car les deux pétreux symétriques du sujet « principal » ont été trouvés plus bas dans le remplissage de l'urne (R02 pour le pétreux gauche, R04 pour le droit).

\section{Bilan provisoire concernant le mausolée III}

41 Si l'on considère que chaque unité de prélèvement constitue un ensemble en soi (en ne tenant donc pas compte des os surnuméraires dont la présence résulte probablement d'une «erreur de collecte»), le nombre total d'individus attestés par des restes osseux brûlés est de 27, à savoir 20 adultes et 7 immatures. On notera que ces derniers sont tous relativement âgés, le plus jeune étant mort à un âge compris entre 7 et 10 ans. Il est néanmoins probable que les dépôts qui n'ont livré qu'une très faible quantité de vestiges (niches 14 ?, 15, 16, 19) seront à mettre en relation avec des lots plus importants. Cela vaut aussi pour le contenu de l'urne III - 35 (inv. 52403) qui semble résulter du curage très partiel d'une aire de crémation.

Tableau 1 - Principaux paramètres de quantification ostéologique enregistrés lors de l'étude des restes humains brûlés issus du mausolée III.

\begin{tabular}{|c|c|c|c|c|c|c|c|c|}
\hline ENSEMBLE & $\begin{array}{l}\text { MASSE } \\
\text { D'OS }\end{array}$ & $\begin{array}{l}\text { NB } \\
\text { FRAGMENTS }\end{array}$ & $\begin{array}{l}\% \\
\text { IDENTIF. }\end{array}$ & $\begin{array}{l}\text { P. } \\
\text { MOY } \\
\text { SAUF } \\
\text { ESQ }\end{array}$ & NMI & ADULTES & IMMATURES & OBSERVATIONS \\
\hline $\begin{array}{l}\text { SEPULCRO } \\
\text { III }\end{array}$ & & & & & & & & \\
\hline III - Urne 1 & 588,3 & 444 & 82,4 & 1,28 & 1 & & 1 & 1 adolescent robuste \\
\hline
\end{tabular}




\begin{tabular}{|c|c|c|c|c|c|c|c|c|}
\hline III - Urne 2 & 1079,7 & 1628 & 72,3 & 0,62 & 1 & 1 & & 1 adulte très gracile \\
\hline III - Urne 22 & 1214,4 & 2175 & 70,6 & 0,51 & 1 & 1 & & 1 adulte $\mathrm{F}$ ? \\
\hline $\begin{array}{l}\text { III - (Busta } \\
\text { A) }\end{array}$ & 976,7 & 1031 & 76,4 & 0,91 & 3 & 2 & 1 & $\begin{array}{l}2 \text { adultes, } 1 \text { Infans II } \\
\text { ou Juvenis }\end{array}$ \\
\hline $\begin{array}{l}\text { III - (Busta } \\
\text { B) }\end{array}$ & 873,4 & 1196 & 70,4 & 0,69 & 2 & 2 & & $\begin{array}{l}1 \text { adulte assez âgé, } 1 \\
\text { adulte d'environ } 25 \\
\text { ans }\end{array}$ \\
\hline III - Niche 7 & 1438,7 & 1485 & 69,2 & 0,90 & 3 & 1 & 2 & $\begin{array}{l}1 \quad \text { adulte, } \\
\text { adolescent, } 1 \text { enfant } \\
7-10 \text { ans }\end{array}$ \\
\hline $\begin{array}{l}\text { III - Niche } \\
14 ? \text { ? (n. « } 5 » \\
\text { Dx) }\end{array}$ & 37,3 & 20 & 51,2 & 1,87 & 1 & 1 & & 1 taille adulte \\
\hline $\begin{array}{l}\text { III - Niche } \\
15\end{array}$ & 56,3 & 14 & 92,9 & 4,02 & 1 & 1 & & 1 taille adulte \\
\hline $\begin{array}{l}\text { III - Niche } \\
16\end{array}$ & 41,3 & 8 & 95,2 & 5,16 & 1 & 1 & & 1 adulte robuste \\
\hline $\begin{array}{ll}\text { III } & \text { Niche } \\
17 \quad \text { (urne } \\
52403)\end{array}$ & 44,5 & 156 & 42,9 & 0,22 & 1 & 1 & & curage de bûcher? \\
\hline $\begin{array}{l}\text { III - Niche } \\
19\end{array}$ & 46,3 & 22 & 83,6 & 2,10 & 1 & 1 & & 1 adulte robuste \\
\hline $\begin{array}{l}\text { III - Niche } \\
20\end{array}$ & 946,3 & 408 & 89,1 & 2,30 & 3 & 2 & 1 & $\begin{array}{l}1 \text { adulte âgé, } 1 \text { taille } \\
\text { adulte, } 1 \text { enfant } \\
10-14 \text { ans }\end{array}$ \\
\hline $\begin{array}{l}\text { III - Niches } \\
8 \text { et } 17\end{array}$ & 1134,1 & 327 & 91,2 & 3,44 & 1 & 1 & & $\begin{array}{l}1 \text { sujet pour deux } \\
\text { niches??? }\end{array}$ \\
\hline $\begin{array}{l}\text { III - Niches } \\
10 \text { et } 11\end{array}$ & 4816,7 & 3512 & 77,3 & 1,31 & 6 & 4 & 2 & $\begin{array}{l}4 \quad \text { adultes, } 1 \\
\text { adolescent, } 1 \text { grand } \\
\text { enfant }\end{array}$ \\
\hline $\begin{array}{l}\text { III - olle US } \\
100\end{array}$ & 447,6 & 243 & 87,6 & 1,81 & 1 & 1 & & $\begin{array}{l}\text { apparemment } 1 \text { sujet } \\
\text { (plusieurs olle???) }\end{array}$ \\
\hline Total & 13741,6 & 12669 & & & 27 & 20 & 7 & \\
\hline
\end{tabular}

Masse totale des os humains brûlés; Nombre de fragments (à l'exception des esquilles); Taux de détermination (en pourcentage de la masse totale); Poids moyen des fragments à l'exclusion des esquilles; Nombre minimal d'individus (adultes et sujets immatures). 
La compréhension des gestes funéraires passera nécessairement par la recherche systématique des liaisons ostéologiques, telle que nous avons pu la pratiquer avec succès pour le mausolée XX. La masse considérable des vestiges que nous avons étudiés (13741,6 g d'os humains brûlés, pour un nombre total de fragments - à l'exclusion des esquilles - de 12669) donne une idée de l'ampleur de la tâche à accomplir. Cette opération ne pourra toutefois être tentée qu'à partir du moment où toutes les urnes du mausolée auront été fouillées et leur contenu étudié. Sur les 31 urnes engagées dans la maçonnerie, une seule a pour l'instant été traitée. Il en reste donc 30, à laquelle il convient d'ajouter l'urne isolée III - 34 (niche 18).

L'étude du mausolée III sera poursuivie en 2014.

\section{BIBLIOGRAPHIE}

Liverani - Spinola 2006 = P. Liverani, G. Spinola, La Necropoli Vaticana lungo la Via Trionfale, Rome, 2006.

Van Andringa et alii 2013 = W. Van Andringa, H. Duday, S. Lepetz (éd.), Mourir à Pompéi : fouille d'un quartier funéraire de la nécropole romaine de Porta Nocera, Rome, 2013 (Collection de l'École française de Rome, 468).

\section{NOTES}

1. Liverani - Spinola 2006.

2. Van Andringa et al. 2013, p. 880.

INDEX

institutions École française de Rome, Musei Vaticani, Université de Bordeaux (PACEA UMR 5199), Soprintendenza speciale per i Beni archeologici di Roma

Mots-clés : nécropole romaine, crémation, anthropologie, épitaphes

\section{AUTEURS}

\section{HENRI DUDAY}

Université Bordeaux 1, PACEA (UMR 5199) - henri.duday[at]u-bordeaux1.fr 


\section{CARLA CALDARINI}

Service d'anthropologie, Soprintendenza speciale per i Beni archeologici di Roma

\section{STEFANIA DI GIANNANTONIO}

Service d'anthropologie, Soprintendenza speciale per i Beni archeologici di Roma

GIANDOMENICO SPINOLA

Musei Vaticani - aca.musei[at]scv.va

\section{LEONARDO DI BLASI}

Musei Vaticani - ac3.musei[at]scv.va

MONICA RICCIARDI

Archéologue 